\title{
Adenosine-sensitive atrial tachycardia originating from the para-Hisian region with a slow conduction zone in the anteroseptal left atrium
}

\author{
Satoshi Sakai ${ }^{1}$, Akihiro Takitsume ${ }^{1}$, Hiroyuki Kawata ${ }^{1}$, Taku Nishida $^{2}$, and Yoshihiko \\ Saito $^{2}$ \\ ${ }^{1}$ Nara Prefecture General Medical Center \\ ${ }^{2}$ Nara Medical University
}

October 13, 2020

\begin{abstract}
Adenosine-sensitive atrial tachycardias (ATs) originating from the para-Hisian region have been reported, and their responsible mechanism is considered to be reentry. As an alternative strategy to ablation at the earliest activaton site (EAS) close to the atrioventricular node, previous reports safely eliminated these ATs at the entrance of the SCZ, indicated by a manifest entrainment-guided strategy, but no report has successfully ablated those ATs using the same strategy in the left atrium. We described a case of adenosine-sensitive AT originating from the para-Hisian region that could be eliminated at a remote site from the EAS indicated by the demonstration of manifest entrainment from the anteroseptal LA.
\end{abstract}

\section{Introduction}

The mechanism responsible for adenosine-sensitive atrial tachycardias (ATs) originating from vicinity of the atrioventricular $(\mathrm{AV})$ node has been considered to be reentry ${ }^{1}$, and they could be ablated safely at the entrance of the slow conduction zone (SCZ) remote from the His bundle region, indicated by a manifest entrainment-guided strategy. ${ }^{2,3}$ In this strategy, manifest entrainment is defined by the orthodromic capture of the electrogram at the earliest activation site (EAS) with a long conduction time by the immediately previous pacing impulse (n-1), and the antidromic capture of other sites by the immediate impulse (n) in one paced beat except for the last entrained beat, equivalent to constant fusion. We described a case of adenosine-sensitive AT originating from the para-Hisian region that could be eliminated at a remote site from the EAS indicated by the demonstration of manifest entrainment from the anteroseptal left atrium (LA).

\section{Case report}

A 73-year-old woman with no history of heart disease developed symptoms of palpitations during hospitalization after surgery for a cerebral aneurysm. The twelve-lead electrocardiogram revealed a long-RP, narrow QRS, regular tachycardia with a heart rate of 132 beats per minute (Figure 1A). After written informed consent was obtained from the patient, an electrophysiological study was performed. Multielectrode catheters were placed at the high right atrium (HRA), His bundle, coronary sinus (CS), and right ventricular apex (RVA). The absence of ventriculoatrial (VA) conduction was inferred by ventricular pacing at several cycle lengths without obtaining retrograde atrial capture (Figure 1B). The supraventricular tachycardia (SVT) was easily induced by burst atrial pacing. The earliest atrial activation was recorded at the His-bundle electrodes (HBE) during the SVT. Ventricular burst pacing during the SVT revealed ventriculoatrial (VA) dissociation. VA linking was not observed during differential atrial overdrive pacing (Figure $1 \mathrm{C})^{4}$ and the 
SVT was reproducibly terminated without AV block by a 4-mg bolus injection of adenosine 5'-triphosphate (Figure 1D). Based on these findings, the SVT was diagnosed as an adenosine-sensitive AT.

The activation map in the RA during the AT revealed a centrifugal pattern. The EAS of the RA (EAS-RA) was located slightly toward the septal portion of the site where the His potentials were recorded (Figure 2A). A local atrial electrogram of the EAS-RA preceded that of the CS proximal (CSp) electrode by 35 ms (Figure $2 \mathrm{~B}$ ). Considering the risk of injuring the AV node by a radiofrequency (RF) application to the EAS-RA, entrainment pacing during the AT with a pacing cycle length (PCL) of 10-20 ms shorter than the tachycardia cycle length (TCL) was performed at various sites in the RA (RA appendage [RAA], high anterolateral RA, high posteroseptal RA, and cavotricuspid isthmus [CTI]) and from the CS electrodes (1 o'clock and 3 o'clock on the MA) to identify the entrance of the SCZ. However, manifest entrainment could not be observed.

Subsequently, the ablation catheter was inserted into the noncoronary aortic sinus (NCS) via a retrograde transaortic approach. Although entrainment pacing from various sites of the NCS was also performed, manifest entrainment could not be demonstrated. The EAS of the NCS (EAS-NCS) with a local potential recorded $41 \mathrm{~ms}$ earlier than that of the CSp electrode (Figure 2B) was located above and slightly posterior to the EAS-RA (Figure 2A). An RF application (25-30 W, 40, 30 seconds) was delivered at the EAS-NCS; however, the AT did not terminate. RF applications (20-25 W, 40) at the EAS-RA where a His potential could not be recorded were also ineffective and were discontinued owing to transient AV block.

Thereafter, activation mapping in the LA performed via a transseptal approach revealed the EAS of the LA (EAS-LA), which was 44ms earlier than that of the CSp electrode (Figure 2B), and was on the opposite side of the EAS-RA (Figure 2A). An RF application (25 W, 40) at the EAS-LA terminated the AT, however, it was interrupted due to transient PR interval prolongation and the AT was still easily inducible after that. Considering the risk of AV block, we hesitated to deliver an additional RF application around the EAS-LA. Thus, entrainment pacing from various sites of the LA were performed considering the possibility that the SCZ of the AT circuit was in the LA. Manifest entrainment could not be observed from the anterior mitral annulus (MA) or base of the left atrial appendage, but was demonstrated from the high anteroseptum (Figure 2A). Entrainment pacing from site A was performed with a PCL of $440 \mathrm{~ms}$ during the AT with a TCL of 455 $\mathrm{ms}$, and the atrial electrograms recorded from the CS were captured antidromically, and those recorded from the HRA and HBE were captured orthodromically with a long conduction interval. Ventricular electrograms recorded from the CS, HBE, and RVA were also orthodromically captured (Figure 3A). From this finding, site A was considered to be proximal to the SCZ, and the entrance of the SCZ was presumed to be located between this pacing site and the EAS-LA (Figure 3B). When the ablation catheter came in contact with Site B, which was $13 \mathrm{~mm}$ superoposterior from the EAS-LA (Figure 2A), and the local elctrogram appeared 8ms later than that of the EAS-LA, the AT became an incessant form. An RF application (25-30 W, 40, 30 seconds) at site B terminated the AT in $3 \mathrm{~s}$ without PR interval prolongation. Thereafter, the AT could no longer be induced. The patient had no recurrence of the tachycardia during a 10-month follow-up period without any antiarrhythmic drugs.

\section{Discussion}

Iesaka and colleagues ${ }^{1}$ first reported adenosine-sensitive AT originating from the vicinity of the AV node and the responsible mechanism was considered to be reentry. Considering the sensitivity to adenosine, calcium channel-dependent tissue such as a retroaortic node or AV ring, which consists of AV node-like structures, is speculated to be involved in the SCZ of the AT circuit ${ }^{5}$. A conventional catheter electrode cannot record the potentials from tissues with Ca channel-dependent conduction, and hence this AT as well as AV nodal reentrant tachycardia (AVNRT) reveals a centrifugal activation on the 3D electroanatomical map. As an alternative strategy to ablation at the EAS representing the exit of the SCZ close to the AV node, previous reports safely eliminated ATs at the entrance of the SCZ that was considered to be located between the EAS and pacing site where manifest entrainment was demonstrated. ${ }^{2,3}$ In this strategy, manifest entrainment is defined by the orthodromic capture of the electrogram at the EAS with a long conduction time by the immediately previous pacing impulse (n-1), and the antidromic capture of the other sites by the immediate 
impulse (n) in one paced beat except for the last entrained beat, equivalent to constant fusion. The reported pacing sites demonstrating manifest entrainment were the RAA, high anterolateral RA, high posteroseptal RA, CTI, NCS ${ }^{6}$, and $\mathrm{MA}^{7}$; however, in our current case, manifest entrainment could not be observed from those sites and could be only from the anteroseptal LA. To our knowledge, the current case is the first report of a case that demonstrated manifest entrainment from the anteroseptal LA and in which successful ablation was achieved at remote site from the EAS, as indicated by a manifest entrainment-guided strategy in the LA. A previous report ${ }^{2}$ showed that the atrial electrograms at the successful ablation site appeared later than that of the EAS by $7^{\sim} 26 \mathrm{~ms}$, and the distance between the successful ablation site and EAS was $7^{\sim} 19$ $\mathrm{mm}$. In our case, the local potential of the last ablation site (site B) had a delay of $8 \mathrm{~ms}$ compared to that of the EAS-LA (Figure 2B), and the distance from the site B to the EAS-LA was $13 \mathrm{~mm}$. These findings suggested that the size of the tachycardia circuit and very long conduction time of the SCZ in this case were comparable to that of the previous report, and the successful ablation site was presumed to be located on the side of the entrance of the SCZ.

Inagaki and colleagues ${ }^{7}$ reported an adenosine-sensitive AT case that demonstrated manifest entrainment from 1 o'clock on the MA. As in their case, if the SCZ is located along the anterior MA, manifest entrainment may be observed from the distal CS electrode. However, if the SCZ is located along the LA septum as in our case, demonstration of manifest entrainment from the CS electrodes seems to be difficult, and entrainment mapping approaching the LA may be required. In their case, the target ablation site was the EAS on the anteroseptum of the LA (10 o'clock on the MA) and was considered to be the exit of the SCZ.

Lyan and colleagues ${ }^{8}$ reported a total of 16 of 68 cases of ATs originating from the para-Hisian region that had ablation in the LA, with only 4 of those, including those with a failed NCS ablation, resulting in the successful elimination of the AT, and ablation in the LA was effective only when the LA activation was earlier than the RA activation as in our case. They also showed the occurrence of transient AV block with 1 unsuccessful case that was ablated in the LA, and in our case as well, an RF application at the EASLA caused transient PR interval prolongation and failed to eliminate the AT. An RF application on the anteroseptum of the atrium near the AV annulus has the potential risk of causing inadvertent AV block even from the LA; therefore, a manifest entrainment guided ablation strategy can be a reasonable option in these cases.

After cessation of the entrainment pacing from site A, a premature atrial complex (PAC) with an early local electrogram recorded by the mapping catheter reset the AT (Figure 3A). The conduction interval between the distal electrode of the mapping catheter and CS distal electrode during entrainment pacing from site A and a PAC was the same (35ms), hence this PAC was presumed to be caused by the mapping catheter. Of note, the last entrained ventricular beat was the second one after the pacing stimulus, indicating a markedly long AV interval; however, a ventricular captured beat by a PAC was the first one after the PAC with a short AV interval despite a shorter coupling interval than the PCL. This finding may be explained by orthodromic capture of the EAS-LA with subsequent capture of the AV node during entrainment pacing (Figure 3B) and capture of the AV node by a PAC before the tachycardia wavefront exited from the SCZ (Figure 3C). During differential atrial overdrive pacing, the last entrained ventricular beat was also the first one with a short AV interval (Figure 1C) due to the antidromic wavefront directly capturing the AV node without propagating to the SCZ because neither the HRA or CS proximal electrode were located proximal to SCZ. This disproportionate AV interval in an adenosine-sensitive AT case was previously reported by Maruyama and colleagues ${ }^{9}$ as a "disproportionate delay". This finding suggested that the SCZ and AV node were in close proximity but not connected, and would provide useful information regarding a challenging differential diagnosis of an adenosine-sensitive AT and AVNRT including a superior type slow pathway ${ }^{10}$.

\section{Conclusion}

We described a case of adenosine-sensitive AT originating from the para-Hisian region with a SCZ in the anteroseptal LA. The SCZ of the AT circuit may be located in the LA even if the earliest atrial activation is observed at the HBE among the electrodes initially placed. Even from the LA, a manifest entrainment guided ablation strategy can be a reasonable option to minimize the potential risk of AV block. 
Figure 1

A: The twelve-lead electrocardiography revealed a long-RP, narrow QRS, regular tachycardia with a heart rate of 132 beats/min. The P-wave morphologies were positive in leads aVL and V1 and biphasic (negative/positive) in leads II, III, and aVF. B: Ventricular burst pacing during the sinus rhythm revealed ventriculoatrial (VA) dissociation. C: The difference in the postpacing ventriculoatrial interval between the high right atrium (HRA) and proximal coronary sinus (CS) entrainment pacing was $44 \mathrm{~ms}>14 \mathrm{~ms}$, indicating the absence of VA linking. D: A 4-mg bolus injection of adenosine5-triphosphate (ATP) terminated the supraventricular tachycardia without atrioventricular block. $\mathrm{d}=$ distal; HBE = His-bundle electrode; RVA $=$ right ventricular apex; $\mathrm{p}=$ proximal; $\mathrm{S}=$ stimulation.

Figure 2

A: Activation maps during the tachycardia. The earliest activation site in the right atrium (EAS-RA), noncoronary aortic sinus (EAS-NCS), and left atrium (EAS-LA) are indicated. The blue star (site A) shows the pacing site where manifest entrainment was observed. The red tag (site B) indicates the successful ablation site. B: The local atrial electrograms of the EAS-RA, EAS-NCS, EAS-LA, site B, and site A during the AT preceded that of the CS proximal electrode by $35 \mathrm{~ms}, 41 \mathrm{~ms}, 44 \mathrm{~ms}, 36 \mathrm{~ms}$ and $32 \mathrm{~ms}$ respectively. $\mathrm{AP}=$ anterior-posterior; $\mathrm{PA}=$ posterior-anterior; $\mathrm{SVC}=$ superior vena cava; $\mathrm{RA}=$ right atrium; $\mathrm{NCS}=$ noncoronary aortic snus; LA = left atrium; $\mathrm{MVA}=$ mitral valve annulus; TVA = tricuspid valve annulus; $\mathrm{MAP}=$ mapping catheter. The other abbreviations are as in Figure 1.

Figure 3

A: Entrainment pacing from site A. The cycle lengths of the tachycardia and pacing are $455 \mathrm{~ms}$ and $440 \mathrm{~ms}$ respectively. The red arrows indicate the orthodromic atrial activation, and the red asterisks indicate the last orthodromic captured electrograms. The blue arrows indicate the antidromic atrial activation, and the blue asterisks indicate the last antidromic captured electrograms. B: Schematic representation of manifest entrainment from site A. The antidromic wavefront (n) captures the coronary sinus (CS), and the orthodromic wavefront of the previous beat (n-1) exits from the slow conduction zone of the reentrant circuit and captures the high right atrium (HRA) and His bundle electrode (HBE), and propagates to the ventricle. C: Schematic representation of a reset of the AT by a premature atrial complex (PAC). A PAC captures the AV node before the tachycardia wavefront exited from the SCZ. AVN = atrioventricular node; $\mathrm{TV}=$ tricuspid valve; $\mathrm{MV}=$ mitral valve. The other abbreviations are as in the previous figures.

1. Iesaka Y, Takahashi A, Goya M, et al. Adenosine-sensitive atrial reentrant tachycardia originating from the atrioventricular nodal transitional area. J Cardiovasc Electrophysiol 1997;8:854-864.

2. Yamabe H, Okumura K, Morihisa K, et al. Demonstration of anatomical reentrant tachycardia circuit in verapamil-sensitive atrial tachycardia originating from the vicinity of the atrio-ventricular node. Heart Rhythm 2012;9:1475-1483.

3. Okumura K, Sasaki S, Kimura M, et al. Usefulness of combined CARTO electroanatomical mapping and manifest entrainment in ablating adenosine triphosphate-sensitive atrial tachycardia originating from the atrioventricular node vicinity. J Arrhythm 2016;32:133-140.

4. Maruyama M, Kobayashi Y, Miyauchi Y, et al. The VA relationship after differential atrial overdrive pacing: a novel tool for the diagnosis of atrial tachycardia in the electrophysiologic laboratory. J Cardiovasc Electrophysiol 2007;18:1127-1133.

5. Bohora S, Lokhandwala Y, Sternick EB, et al. Reappraisal and new observations on atrial tachycardia ablated from the non-coronary aortic sinus of Valsalva. Europace 2018;20:124-133.

6. Satoshi S, Hajime F, Takehito $\mathrm{N}$ et al. Adenosine-sensitive atrial tachycardia originating from the para-Hisian region with the entrance of a slow conduction zone at the noncoronary aortic sinus. HeartRhythm Case Rep 2020;6:448-452.

7. Inagaki D, Hojo R, Fukamizu S, et al. Adenosine-sensitive atrial tachycardia originating from the anterior mitral annulus. HeartRhythm Case Rep 2018;4:542-544.

8. Evgeny L, Mauro T, Alexey T, et al. Comparison of strategies for catheter ablation of focal atrial 
tachycardia originating near the His bundle region Heart Rhythm 2017;14:998-1005.

9. Mitsunori M, Seiji T, Wataru S. Disproportionate delay in ventricula capture during atrial overdrive pacing in along RP supra ventricular tachycardia: What is the mechanism? HeartRhythm2016;13:1563-1564

10. Kiyoshi O, Yasutoshi N, Hiroshi T. Superior type of atypical AV nodal reentrant tachycardia: incidence, characteristics, and effect of slow pathway ablation. Pacing Clin Electrophysiol. 2008;31:998-1009.

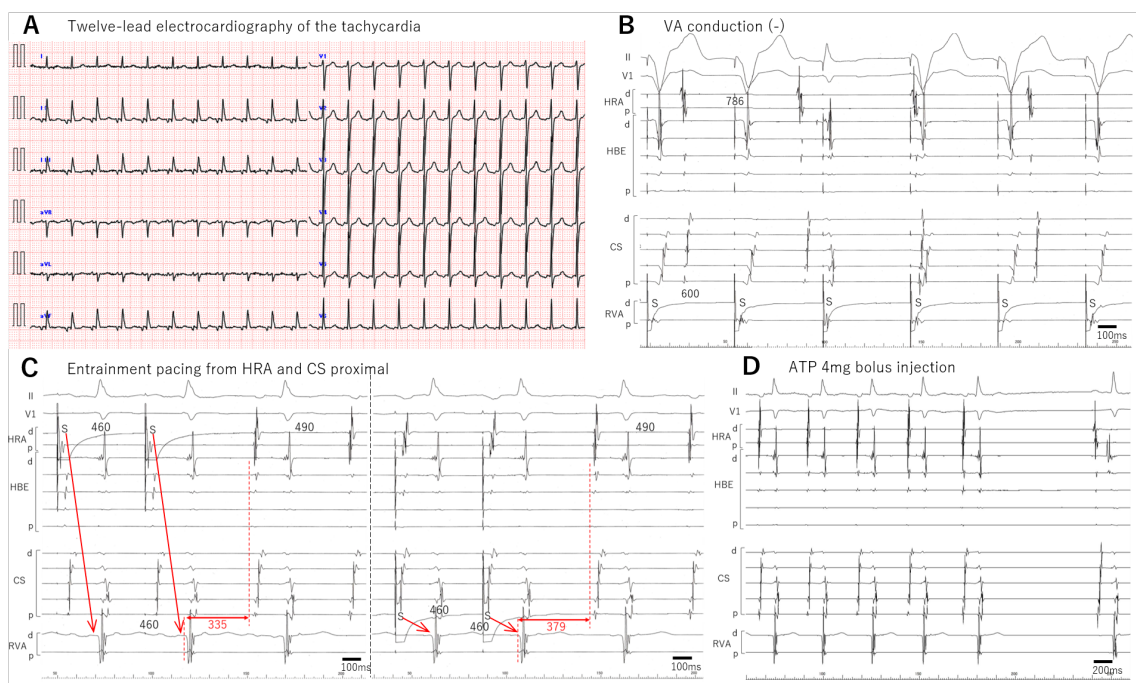

A

AP

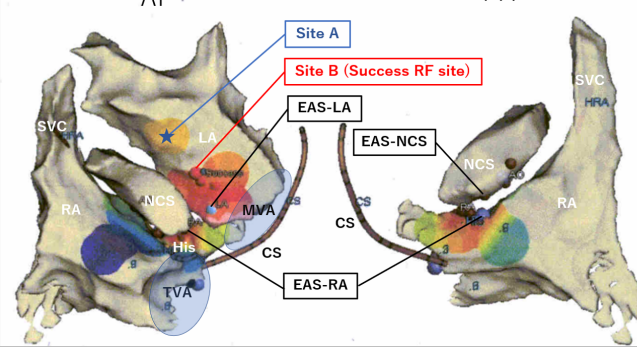

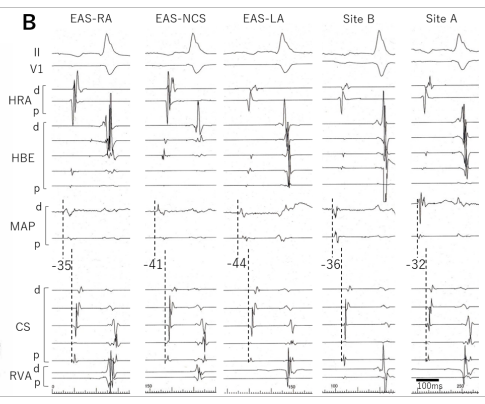




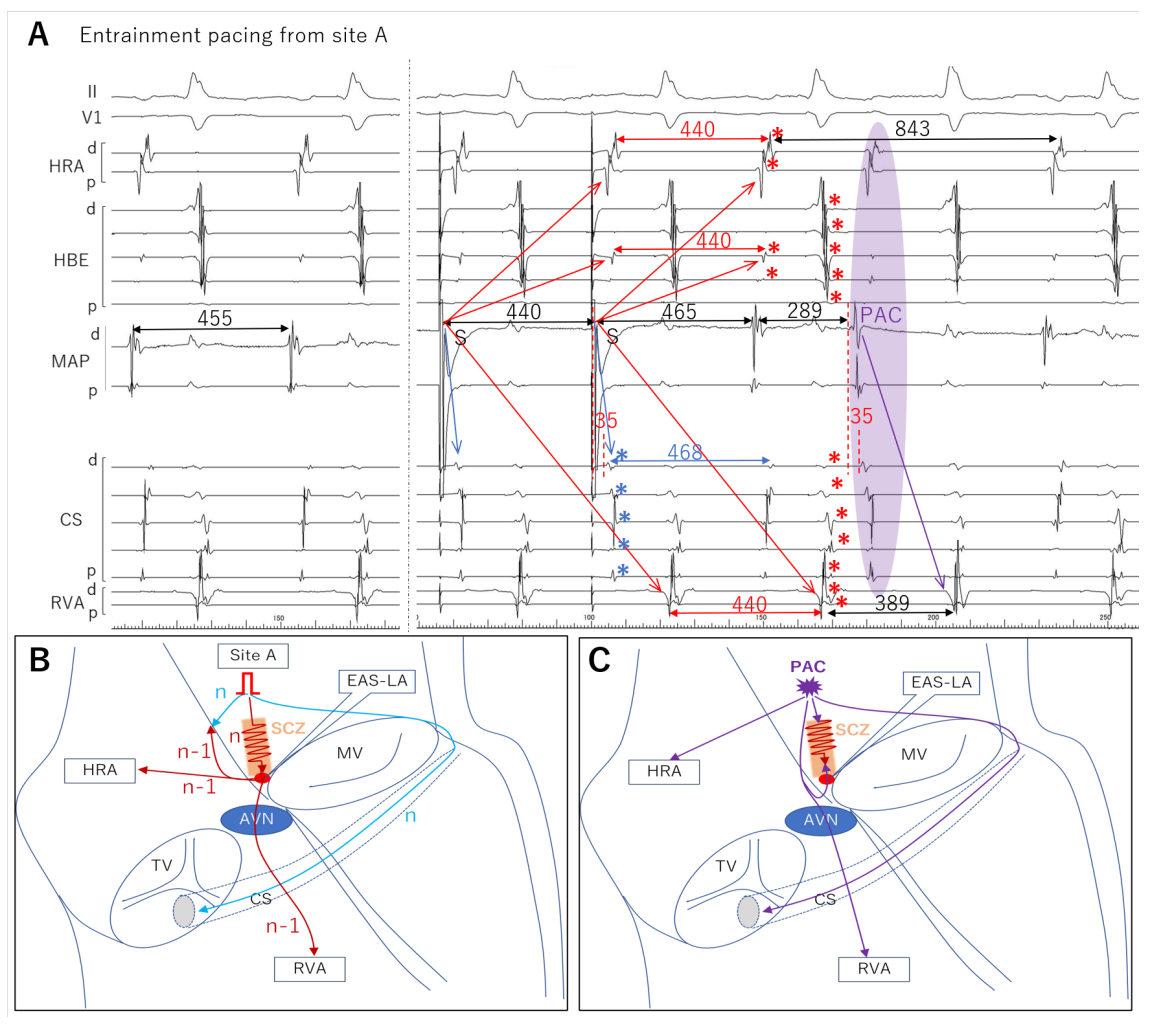

